\title{
Liver Rhabdomyosarcoma
}

National Cancer Institute

\section{Source}

National Cancer Institute. Liver Rhabdomyosarcoma. NCI Thesaurus. Code C5834.

A malignant mesenchymal tumor with skeletal muscle differentiation affecting the liver. 